\title{
Influencia de la estructura de aditivos basados en policarboxilato sobre el comportamiento reológico de pastas de cemento
}

\author{
Effect of polycarboxylate admixture structure \\ on cement paste rheology
}

\author{
M. M. Alonso(*), M. Palacios ${ }^{(*)}$, F. Puertas $^{(*)}$, A. G. de la Torre(**) y M. A. G. Aranda(**)
}

Recepción/Received: 19-VII-06

Aceptación/Accepted: 5-XII-06

\section{RESUMEN}

El objetivo de este trabajo ha sido estudiar el efecto de las diferencias estructurales de cuatro aditivos basados en policarboxilatos y poliéteres sobre las propiedades reológicas de pastas de cemento con diferente composición química, mineralógica y con distintas adiciones activas (CEM I 42,5 R, CEM I 52,5 R, CEM I 52,5 N/SR, CEM II/AV 42,5R, CEM II/ B-L 32,5 R, CEM III/B 32,5R, BL I 52,5R y CAC - Norma EN 197-1:2000). Los resultados obtenidos sobre la fluidez de la pasta en el ensayo del "Minislump" coinciden con la evolución de los valores de los parámetros reológicos (esfuerzo de cizalladura y viscosidad plástica) determinados a través de un viscosímetro rotacional. Las características estructurales de los aditivos que más afectan al efecto fluidificante son el contenido de grupos carboxilatos (GC) y de grupos poliéteres (GE). En relación con las características y composición de los cementos, son factores fundamentales, la finura y las relaciones $\mathrm{C}_{3} \mathrm{~A} /$ sulfato cálcico y $\mathrm{C}_{3} \mathrm{~S} / \mathrm{C}_{3} \mathrm{~A}$. Así, las pastas de CEM I 52,5N/SR son las que presentan una mayor modificación de los parámetros reológicos, mientras que las de BL I 52,5R son las que se ven modificadas en menor medida. Las pastas de CEM III/B 32,5R, con escoria granulada de horno alto, son las que presentan mayores incrementos de la fluidez más acusados. Por último, el efecto fluidificante del aditivo es mucho más marcado en cementos de aluminato de calcio aunque presenta acusadas pérdidas de fluidez con el tiempo.

Palabras clave: cemento Portland, superplastificantes reología, adición, compatibilidad.

\section{SUMMARY}

The purpose of the present study was to analyze the effect of the structural differences in four polycarboxylate and polyether admixtures on the rheological properties of cement pastes with different chemical and mineralogical compositions and different active additions (CEM I $42.5 R$, CEM I 52.5 R, CEM I 52.5 N/SR, CEM II/AV 42.5R, CEM II/B-L 32.5 R, CEM III/B 32.5R, BL I 52.5R and CAC-European standard EN 197-1:2000). The results of the minislump test concurred with the variations observed in the values of the rheological parameters (shear stress and plastic viscosity). The structural characteristic of the admixtures found to play the most prominent role in their fluidizing effect was the proportion of carboxylate (CG) and polyether (EG) group components. In cements characteristics such as fineness and the $C_{3} A$ /calcium sulphate and $C_{3} S / C_{3} A$ ratios were also observed to be essential to admixture effectiveness. In this regard, the rheological parameters varied most widely in CEM I 52.5N/SR pastes and least in BL I 52.5R cement pastes. Of the additioned cements, the CEM III/B $32.5 R$ pastes, which contained granulated blast furnace slag, showed the highest rises in flowability. Finally, the fluidizing effect of polycarboxylate superplasticizers was much more intense in calcium aluminate cements, although flowability declined rapidly in this material.

Keywords: Portland cement, superplasticizers, rheology, addition, compatibility.

(*) Instituto de Ciencias de la Construcción Eduardo Torroja (CSIC), Madrid (España).

(**) Departamento de Química Inorgánica, Cristalografía y Mineralogía, Univ. de Málaga, Málaga (España). 


\section{INTRODUCCIÓN}

En los últimos años, el empleo y desarrollo de aditivos superplastificantes basados en policarboxilato ha sido determinante para la preparación de hormigones autocompactantes y de altas prestaciones $(1,2)$. Estos aditivos inducen una disminución del contenido de agua del hormigón de hasta el $40 \%$, siendo dicha reducción superior a la que originan los aditivos superplastificantes convencionales (basados en lignosulfonatos, melaminas, naftalenos). El empleo de aditivos basados en policarboxilato, además de mejorar la trabajabilidad y puesta en obra del hormigón, produce una disminución de su porosidad y, por lo tanto, una mejora de sus propiedades mecánicas y durables.

Estos aditivos basados en policarboxilato se caracterizan por presentar una estructura tipo "peine" (3) con una cadena hidrocarbonada lineal principal y cadenas laterales constituidas por grupos carboxilato y grupos éteres. Estos aditivos se adsorben a través de los grupos carboxilato sobre las partículas de cemento y originan su dispersión como consecuencia de la repulsión electrostática y fundamentalmente repulsión estérica, asociada a las largas cadenas de grupos éteres $(4,5)$. Recientemente, se han desarrollado aditivos basados en policarboxilatos con estructuras modificadas, que contienen cadenas principales más cortas y cadenas laterales de poliéteres más largas (6). Numerosos investigadores (7-9) han concluido que las diferentes estructuras de estos aditivos, su peso molecular, así como su distribución de pesos moleculares, afectan directamente a la fluidez, a la resistencia a la segregación y al comportamiento reológico de las pastas, morteros y hormigones de cemento.

En opinión de algunos autores $(10,11)$, cuanto más corta sea la cadena principal y más largas y numerosas sean las cadenas laterales de éteres, mayor y más duradera será la fluidez inducida por el aditivo. Sin embargo, Winnefeld et al. (12) indicaron que en el caso de aditivos que están constituidos por cadenas laterales de poliéteres cortas, el efecto fluidificante inducido no depende de la densidad de dichas cadenas. Por el contrario, para longitudes de cadena laterales media o larga cuanto menor es la densidad de cadenas laterales, mayor es el incremento de la fluidez que el aditivo origina sobre las pastas de cemento. El peso molecular de los polímeros también tiene una influencia destacada en el comportamiento de los aditivos, de modo que cuanto mayor sea el peso molecular, mayor es la adsorción y mayor la fluidez que inducen (8). Sin embargo, no sólo hay que considerar el peso molecular de los aditivos, sino además su distribución de pesos moleculares observándose que cuanto menor sea éste, mayor será el efecto de los aditivos sobre la fluidez (13). Finalmente, y al contrario de lo que sucede en el caso de los aditivos derivados de naftaleno, en

\section{INTRODUCTION}

The use and development of polycarboxylate superplasticizers (also known as high-range water reducing admixtures) has been instrumental to the preparation of selfcompacting and high performance concretes $(1,2)$. With reductions of up to $40 \%$ of the water in concrete, such admixtures are much more effective than conventional superplasticizers (based on lignosulphonates, melamines and naphthalenes). In addition to improving concrete workability during placement, polycarboxylate admixtures reduce porosity and therefore enhance mechanical strength and durability.

Such admixtures are characterized by a "comb-type" structure (3), in which the backbone is a linear hydrocarbonate chain with carboxylate groups and ether lateral chains forming the teeth. Their adsorption on cement particles, mediated by their carboxylate groups, induces cement grain dispersion as a result of both the electrostatic and especially the steric repulsion generated by the long length of ether group chains $(4,5)$. Modified polycarboxylate admixtures have recently been developed with shorter backbones and longer polyether lateral chains (6). Many researchers (7-9) have concluded that the differences in the structure, molecular weight and molecular weight distribution of these admixtures have a direct effect on cement paste, mortar and concrete flowability, resistance to segregation and rheological behaviour.

Some authors $(10,11)$ believe that the shorter the backbone and the longer and more numerous the ether lateral chains, the greater and longer-lasting is the flowability induced. According to Winnefeld et al. (12), in admixtures consisting in short lateral polyether chains, the fluidizing effect induced does not depend on the density of these chains. In admixtures with medium or long lateral chains, on the contrary, the smaller the number of lateral chains, the greater is the fluidity generated by the admixture in cement pastes. The molecular weight of these polymers also has a substantial effect on their behaviour; polymers with greater molecular weight are adsorbed in higher proportions and induce more fluidity in the system (8). But admixture molecular weight is not the only consideration: account must also be taken of the distribution of molecular weight, for the lower the distribution of molecular weight the greater is the admixture effect on fluidity (13). Finally and contrary to the mechanisms observed in naphthalene admixtures, the nature of the counter-ion in polycarboxylate admixtures does not modify their fluidizing effect (14). The variety 
los aditivos basados en policarboxilato, la naturaleza del contraión que contienen no modifica su efecto fluidificante (14). Son tantas las posibles formulaciones y estructuras moleculares que pueden adoptar estos nuevos aditivos que no se ha establecido aún cuál es la relación existente entre el número de grupos carboxilato/grupos poliéteres de los mismos y las propiedades reológicas y dispersivas que inducen en las pastas y hormigones.

Numerosos autores concluyen que el efecto de los aditivos basados en policarboxilato depende de factores asociados al propio cemento como el contenido en $\mathrm{C}_{3} \mathrm{~A}$, el tipo y contenido del sulfato cálcico utilizado como regulador de fraguado (15), álcalis del clínker (13), la superficie específica y distribución de tamaño de partícula del cemento (16) y el tipo de adición que contiene.

En torno a la composición del cemento, algunos autores indican que los aditivos basados en policarboxilato se adsorben preferiblemente sobre la fase $C_{3} A$, aunque dicha adsorción disminuye en presencia de yeso (17). En este sentido, Flatt y Houst (14) concluyen que el incremento del contenido de sulfatos del cemento favorece la formación de etringita por encima de la formación de una fase órgano-mineral que contiene el aditivo intercalado. Maragotto et al. (18) indicaron que el efecto fluidificante de los aditivos basados en policarboxilato disminuye en cementos con elevados contenidos de álcalis solubles. Con respecto a la finura del cemento, numerosos trabajos han demostrado que cuanto mayor es la superficie específica del cemento y, por lo tanto, mayor es su finura, mayor es la adsorción de un aditivo superplastificante (19). Sin embargo, no sólo debe considerarse la superficie del cemento, sino también su distribución de tamaño de partícula, debido a que las diferentes fracciones granulométricas pueden diferir en su composición $y$, consecuentemente, en la adsorción de los aditivos sobre dichas fracciones.

Finalmente, existen muy pocos trabajos que expliquen la compatibilidad de los aditivos basados en policarboxilato con cementos que contengan adiciones de diferente naturaleza. En este sentido, Chongzhi et al. (20) indicaron que la incorporación de adiciones de cenizas volantes y humo de sílice mejora la compatibilidad aditivocemento, obteniéndose los mejores incrementos de fluidez cuando el contenido de adición es superior al $30 \%$ con respecto a la masa de cemento.

En general, es bastante conocido el efecto que inducen los aditivos superplastificantes convencionales sobre el comportamiento reológico en pastas, morteros y hormigones (21), sin embargo, existen muchas incógnitas en torno al comportamiento de los mismos con cementos de diferente composición mineralógica y adiciones. of possible formulations and molecular structures that may be adopted by these new admixtures is so wide that no relationship has yet been established between the carboxylate group/polyether group ratio and the rheological and dispersive properties induced in pastes and concretes.

Many authors maintain that the effect of polycarboxylate admixtures depends on factors associated with the cement itself, such as $C_{3} A$ content, type and content of calcium sulphate used as a setting regulator (15), clinker alkalis (13), specific surface, particle size distribution (16) and type of addition.

Concerning to the cement composition, some researchers report that polycarboxylate admixtures are more readily adsorbed on the $C_{3} A$ phase, but such adsorption declines in the presence of gypsum (17). In this regard, Flatt and Houst (14) conclude that the increase in cement sulphate content favours the formation of ettringite over the generation of an organic-mineral phase containing the interspersed admixture. Maragotto et al. (18) report that the fluidizing effect of polycarboxylate admixtures declines in cements with high soluble alkali contents. Many studies have shown that the larger the specific surface of the cement and consequently the greater its fineness, the higher is the superplasticizer adsorption (19). Nonetheless, the specific surface of the cement should not be considered alone, but in conjunction with particle size distribution, due to the fractions may differ in composition and consequently in the degree of admixture adsorption.

Finally, very few studies have explored the compatibility between polycarboxylate admixtures and cements containing different types of additions. In this regard, Chongzhi et al. (20) found that the inclusion of fly ash and silica fume additions enhanced admixture-cement compatibility, as the highest rises in flowability were recorded for cements with high proportions of additions (over $30 \%$ by cement mass).

The effect of conventional superplasticizers on paste, mortar and concrete rheology is generally well understood (21). There are still many aspects of their behaviour in blended cements with different mineralogical compositions, however, that remain to be explained. 
Por lo tanto, el objetivo de este trabajo es conocer cómo afectan las características estructurales de aditivos PC y PE sobre el comportamiento reológico de cementos normalizados con distinta composición mineralógica y adiciones.

\section{EXPERIMENTAL}

\subsection{Materiales}

Se utilizaron ocho cementos Portland normalizados (Norma EN 197-1:2000). La Tabla 1 muestra la composición química de los diferentes cementos, así como su superficie específica determinada por el método Blaine (UNE-EN 196-6). En la Tabla 2 se muestra la composición mineralógica obtenida con el método de Rietveld $(22,23)$, incluyendo el contenido de material amorfo en algunas de las muestras (24). Las descripciones cristalográficas y los protocolos utilizados para realizar los ajustes de los difractogramas con la metodología de Rietveld han sido los establecidos en la bibliografía (25). La Tabla 3 y la Figura 1 muestran las características físico-químicas y las estructuras de los cuatro aditivos superplastificantes basados en policarboxilato y poliéteres utilizados, respectivamente. La estimación de los grupos funcionales se realizó siguiendo la metodología descrita en la bibliografía $(26,27)$.

\subsection{Ensayos realizados}

\subsubsection{Determinación del comportamiento reológico}

Para la realización de los ensayos reológicos se prepararon pastas de los diferentes cementos con una relación
The purpose of the present study, then, is to ascertain how the structural characteristics of PC and PE admixtures affect the rheology of a series of standardized additioned cements with different mineralogical compositions, and mineral additions.

\section{EXPERIMENTAL}

\subsection{Materials}

Eight standard Portland cements were used (European standard EN 197.1:2000), whose chemical composition and specific surface as determined with the Blaine method (Spanish/European standard UNE-EN 196-6) are given in Table 1. Table 2 shows the mineralogical composition found by Rietveld analysis $(22,23)$, along with the amorphous material content in some samples (24). The crystallographic descriptions and protocols used for Rietveld refinement of the diffractograms were as described in the literature (25). Table 3 and Figure 1 respectively show the physical-chemical characteristics and structures of the four polycarboxylate/polyether superplasticizer admixtures used. The functional groups were estimated using the methodology described in the literature $(26,27)$.

\subsection{Tests conducted}

\subsubsection{Determination of rheological behaviour}

The rheological tests were conducted on pastes made with a water/cement ratio of 0.4. Due to their greater

Tabla 1 / Table 1

Composición química (\% peso) y superficie específica Blaine de los cementos utilizados Chemical composition (\% by weight) and Blaine specific surface of the cements used

\begin{tabular}{|c|c|c|c|c|c|c|c|c|}
\hline & Cem I 42.5R & Cem I 52.5R & Cem I 52.5N/SR & Cem II/AV 42.5R & Cem II/ B-L 32.5R & Cem III/B 32.5R & BL I 52.5R & CAC \\
\hline P.F & 3.38 & 1.92 & 2.56 & 1.77 & 10.59 & 0.6 & 3.43 & 0.22 \\
\hline $\mathrm{SiO}_{2}$ & 21.13 & 19.08 & 20.09 & 24.81 & 19.25 & 29.5 & 20.94 & 3.96 \\
\hline $\mathrm{Al}_{2} \mathrm{O}_{3}$ & 4.16 & 4.63 & 6.17 & 6.51 & 6.7 & 11.39 & 4.30 & 44.21 \\
\hline $\mathrm{Fe}_{2} \mathrm{O}_{2}$ & 3.8 & 4.3 & 2.30 & 3.81 & 1 & 1.73 & 0.20 & 15.56 \\
\hline $\mathrm{CaO}$ & 63.94 & 62.54 & 62.49 & 58.73 & 79.14 & 47.26 & 66.88 & 38.9 \\
\hline $\mathrm{MgO}$ & 0.13 & 0.2 & 0.18 & 0.16 & 0.46 & 5.67 & 0.24 & 0.04 \\
\hline Sulfatos / Sulphates & 1.03 & 3.35 & 2.81 & 3.07 & 2.8 & 1.11 & 3.12 & - \\
\hline Sulfuros / Sulphides & - & - & - & - & - & 1.04 & - & - \\
\hline RI & 2.26 & 0.55 & 0.73 & 8.82 & 2.05 & 0.44 & 0.11 & 2.26 \\
\hline $\mathrm{Na}_{2} \mathrm{O}$ & 0.25 & 0.32 & 0.27 & 0.27 & 0.26 & 0.27 & 0.20 & 0.26 \\
\hline $\mathrm{K}_{2} \mathrm{O}$ & 0.74 & 0.8 & 0.73 & 0.56 & 0.65 & 0.71 & 0.60 & 0.55 \\
\hline CaO libre / $\mathrm{CaO}$ free & 1.28 & 1.58 & 0.5 & 1.34 & 1.35 & 0.58 & 1.29 & 0.38 \\
\hline Blaine $\left(\mathrm{m}^{2} / \mathrm{kg}\right)$ & 386.7 & 481.5 & 397.9 & 387.6 & 438.7 & 383.1 & 368.0 & 342.8 \\
\hline
\end{tabular}

P.F. Pérdida a fuego / L.O.I. Loss on ignition.

RI Residuo insoluble / IR Insoluble residue. 
Tabla 2 / Table 2

Composición mineralógica de los cementos utilizados (\% peso) Mineralogical composition of the cements used (\% weight)

\begin{tabular}{|c|c|c|c|c|c|c|c|c|}
\hline & Cem I 42.5R & Cem I 52.5R & Cem I 52.5N/SR & Cem II/AV 42.5R & Cem II/ B-L 32.5R & Cem III/B 32.5R & BL I 52.5R & CAC \\
\hline $\mathrm{C}_{3} \mathrm{~S}$ & $62.5(2)$ & $60.0(2)$ & $66.4(2)$ & $43.0(2)$ & $45.4(2)$ & $21.5(2)$ & $65.9(2)$ & - \\
\hline $\mathrm{C}_{2} \mathrm{~S}$ & $8.3(5)$ & $13.2(5)$ & $11.0(6)$ & $6.7(5)$ & $10.0(4)$ & $0.7(1)$ & $14.3(5)$ & - \\
\hline $\mathrm{C}_{3} \mathrm{~A}$ & $4.0(2)$ & $4.7(2)$ & $0.5(1)$ & $2.7(2)$ & $3.6(1)$ & $1.1(1)$ & $7.7(2)$ & - \\
\hline $\mathrm{C}_{4} \mathrm{AF}$ & $11.9(2)$ & $12.3(2)$ & $14.1(3)$ & $7.8(2)$ & $8.5(2)$ & $3.1(1)$ & - & $3.6(1)$ \\
\hline CA & - & - & - & - & - & - & - & $74.2(2)$ \\
\hline $\mathrm{C}_{12} \mathrm{~A}_{7}$ & - & - & - & - & - & - & - & $1.9(1)$ \\
\hline $\mathrm{FeO}$ & - & - & - & - & - & - & - & $4.0(1)$ \\
\hline $\mathrm{CaCO}_{3}$ & $3.7(2)$ & $2.3(2)$ & $3.5(2)$ & - & $26.7(2)$ & - & $6.5(3)$ & - \\
\hline $\mathrm{CaSO}_{4} \cdot 2 \mathrm{H}_{2} \mathrm{O}$ & $5.4(1)$ & $2.2(1)$ & $0.9(1)$ & $2.5(1)$ & $1.4(1)$ & & $4.8(2)$ & - \\
\hline $\mathrm{CaSO}_{4} \cdot 1 / 2 \mathrm{H}_{2} \mathrm{O}$ & $1.3(1)$ & $1.6(1)$ & $1.8(2)$ & & $2.1(2)$ & & & \\
\hline $\mathrm{CaSO}_{4}$ & $0.6(1)$ & $0.8(1)$ & $0.6(1)$ & & $1.1(1)$ & $0.6(1)$ & & \\
\hline Sulfatos alcalinos & $1.7(1)$ & $1.4(1)$ & $1.2(2)$ & $1.1(1)$ & $1.2(1)$ & $0.8(1)$ & $0.8(1)$ & \\
\hline \multicolumn{9}{|l|}{ Alkaline sulphates } \\
\hline $\mathrm{SiO}_{2}$ & & & - & $0.4(1)$ & - & & - & - \\
\hline Otros / Other & $0.5(1)$ & $1.5(1)$ & & $0.9(1)$ & & $0.3(1)$ & - & $16.3(4)$ \\
\hline $\begin{array}{l}\text { Amorfo } \\
\text { Amorphous }\end{array}$ & $*$ & * & * & $34.9(6)^{* *}$ & * & $71.9(6)^{* *}$ & * & * \\
\hline
\end{tabular}

* No se ha cuantificado / Not quantified.

** Análisis cuantitativo del material amorfo según (25) / Quantitative analysis of amorphous material as per (25).

Tabla 3 / Table 3

Características físicas y químicas de los aditivos Admixture physical and chemical characteristics

\begin{tabular}{|l|ccc|}
\hline & SP1 & SP2 & SP3 \\
\hline Contenido en sólidos (\%) (UNE-EN 480-8) / Solids content (\%) (UNE-EN 480-8) & 40.68 & 39.94 & 39.67 \\
\hline Viscosidad rotacional (mPa·s) / Rotational viscosity (mPa.s) & 126.2 & 432.86 & 865.02 \\
\hline Porcentaje de grupos carboxilatos (GC) / Percentage of carboxylate groups (CG) & 32.8 & 16.7 & 818.0 \\
\hline Porcentaje de grupos poliéteres (GE) / Percentage of polyether groups (EG) & 50 & 69.4 & 78.3 \\
\hline GC/GE / CG/EG & 0.656 & 0.240 & 0.112 \\
\hline Contenido en Na (ppm) / Na content (ppm) & 25500 & 13200 & 0.057 \\
\hline
\end{tabular}

agua/cemento fijada en 0,4 . Sin embargo, se pudo observar que los cementos CEM I 52,5R y BL I 52,5R, debido a su mayor finura, requerían más agua. Por su parte el CAC requirió una menor relación a/c. La Tabla 4 muestra las relaciones agua/cemento empleadas.

El comportamiento reológico de las pastas de cemento se caracterizó mediante el ensayo de "Minislump" y mediante un viscosímetro rotacional.

Ensayo de "Minislump": Los diferentes aditivos basados en policarboxilatos y poliéteres se incorporaron junto con el agua de amasado en una dosificación entre el 0,1 y $0,3 \%$. Las pastas se mezclaron durante 3 minutos en una amasadora, al cabo de los cuales se introdujeron en un molde troncocónico de dimensiones $190 \times 381$ × 572 mm. fineness, however, cements CEM I 52.5R and BL I $52.5 R$ required more water. $C A C$, in turn, called for a smaller $w / c$ ratio. Table 4 shows the water/cement ratios used.

Cement paste rheology was studied with the "Minislump" test and a rotational viscometer.

"Minislump" test: The polycarboxylate and polyether admixtures were added to the cement with the water at dosages of from 0.1 to $0.3 \%$. After mixing for 3 minutes in a laboratory mixer, the pastes were poured into truncated cone moulds measuring $190 \times 381 \times 572 \mathrm{~mm}$ The mould was subsequently removed from the samples set 
Tabla 4 / Table 4

Relaciones agua/cemento de las pastas

Paste water/cement ratios

\begin{tabular}{|l|cccccccc|}
\hline $\begin{array}{l}\text { Tipo de cemento } \\
\text { Cement }\end{array}$ & Cem I 42,5R & Cem I 52,5R & $\begin{array}{c}\text { Cem I } \\
\mathbf{5 2 , 5 N / S R}\end{array}$ & $\begin{array}{c}\text { Cem II } \\
\text { /AV 42,5R }\end{array}$ & $\begin{array}{c}\text { Cem II } \\
\text { /BL 32,5R }\end{array}$ & $\begin{array}{c}\text { Cem III } \\
\text { /B 32,5R }\end{array}$ & BL I 52,5R & CAC \\
\hline $\begin{array}{l}\text { Relación agua/cemento } \\
\text { Water/cement ratio }\end{array}$ & 0.40 & 0.45 & 0.40 & 0.40 & 0.40 & 0.40 & 0.50 & 0.35 \\
\hline
\end{tabular}

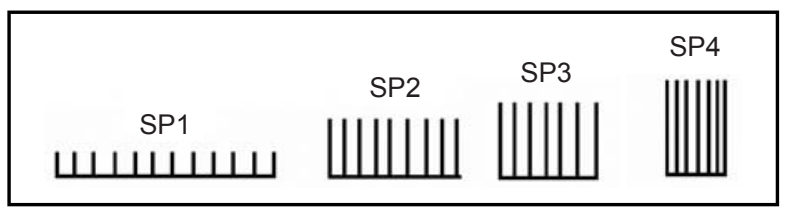

Figura 1. Estructura de los aditivos.

Figure 1. Admixtures structures.

Posteriormente, el molde se retiró y se dieron 10 golpes en una mesa de sacudidas. Se midió el diámetro de la pasta en 4 direcciones y el valor final fue su media aritmética. Este ensayo se realizó a diferentes tiempos desde el amasado de las pastas: $5,15,30$ y 60 minutos (28).

Ensayos reológicos mediante un viscosímetro rotacional: se determinaron los parámetros reológicos (esfuerzo de cizalla umbral y viscosidad plástica) de las pastas de cemento empleando un viscosímetro Haake Rheowin Pro RV1 con un rotor cilíndrico con acanaladuras.

Se prepararon pastas de cemento mezclando durante 3 minutos y mediante un agitador de aspas, $100 \mathrm{~g}$ de cemento con la cantidad de líquido determinada para obtener la relación líquido/sólido indicada en la Tabla 4. Junto con el agua de amasado se incorporaron dosificaciones de aditivo comprendidas entre el 0 y el $0,3 \%$ en peso de cemento.

Las pastas se sometieron a un ensayo reológico que consistió en una pre-agitación de $100 \mathrm{~s}^{-1}$ durante 1 minuto, posteriormente la velocidad del rotor se disminuyó a $10 \mathrm{~s}^{-1}$, incrementándose a continuación a $100 \mathrm{~s}^{-1}$ en 2,5 minutos, disminuyendo finalmente en el mismo intervalo de tiempo hasta $10 \mathrm{~s}^{-1}$.

\subsubsection{Determinación del potencial zeta de los cementos}

Se prepararon suspensiones de los distintos cementos, así como de las adiciones (ceniza volante, escoria y caliza) en agua. Para ello se pesaron $40 \mathrm{~g}$ de los cementos y de las adiciones tamizados por debajo de $20 \mu \mathrm{m}$ y $140 \mathrm{~g}$ de agua y se mezclaron durante 15 minutos con un agitador magnético. Posteriormente, las suspensiones se sometieron durante 5 minutos en un equipo de ultrasonidos Bandelin Sonoplus UW 2200 e inmediatamente sobre ellas se determinó el potencial zeta, empleando para ello un equipo Acoustosizer IIs de Colloidall dynamics. on a flow table that was raised and dropped ten times. The paste diameter was measured in four directions and the arithmetic mean taken as the final value. The test was conducted 5, 15, 30 and 60 minutes after the pastes were mixed (28).

Rotational viscometer tests: paste rheological parameters (shear stress and plastic viscosity) were determined with a Haake Rheowin Pro RV1 viscometer having a serrated coaxial rotor.

Cement pastes $-100 \mathrm{~g}$ of cement with the amount of liquid needed to obtain the liquid/solid ratio specified in Table 4- were stirred for three minutes with a blade stirrer. Admixture dosages ranging from 0 to $0.3 \%$ by weight of cement were added.

The pastes were subjected to a rheological test consisting in pre-shear at $100 \mathrm{~s}^{-1}$ for one minute, after which the rotor speed was reduced to $10 \mathrm{~s}^{-1}$, then increased to $100 \mathrm{~s}^{-1}$ over 2.5 minutes and finally reduced over the same period of time to $10 \mathrm{~s}^{-1}$.

\subsubsection{Determination of cement zeta-potential}

Cements and additions (fly ash, slag and limestone) suspensions were prepared. In this operation, $40 \mathrm{~g}$ of the cements and additions, sieved to under $20 \mu \mathrm{m}$, and $140 \mathrm{~g}$ of water were mixed for 15 minutes with a magnetic stirrer. The suspensions were subsequently subjected during 5 minutes to ultrasound with a Bendelin Sonoplus UW 2200 sonicator and the zeta-potential was immediately determined with a Colloidall Dynamics Acoustosizer IIs analyzer. 


\section{RESULTADOS}

\subsection{Determinación del comportamiento reológico}

\subsubsection{Ensayo de "Minislump"}

Los resultados del ensayo del "Minislump" se presentan en la Figura 2. El análisis de los diferentes gráficos muestra que los aditivos SP1, SP2 y SP3 inducen un mayor efecto fluidificante que el aditivo SP4.

Se observa que los aditivos SP1, SP2 y SP3 incrementan en torno al $16-40 \%$ la fluidez de las pastas de cemento CEM I 42.5R, CEM I 52.5NSR, CEM I 52.5R, CEM II/AV 42.5R y CEM II/BL 32.5R cuando la dosificación de aditivo empleada es del 0,3\%; sin embargo, la fluidez de las pastas de BL I 52.5R sólo se ve incrementada con la incorporación del aditivo SP3. Por otra parte, las pastas de cemento de CEM III/B 32.5R y CAC experimentan segregación cuando la dosificación empleada de los aditivos SP1, SP2 y SP3 es del $0,3 \%$. En el caso de las pastas del cemento CEM III/B $32.5 \mathrm{R}$ la incorporación de $0,1 \%$ de dichos aditivos incrementa su fluidez hasta un $53 \%$, mientras que dicho incremento es de hasta el $90 \%$ en las pastas de cemento tipo $\mathrm{CAC}$, aunque estas últimas pierden inmediatamente su fluidez cuando se incorporan los aditivos SP1 y SP2.

Por el contrario, el aditivo SP4 apenas modifica la fluidez de las pastas de cemento de CEM I 42.5R, CEM I 52.5R, CEM I 52.5NSR, CEM II/AV 42.5R, CEM II/BL 32.5R y BL I $52.5 R$, y si bien incrementa la fluidez de las pastas de CAC y de CEM III/B 32.5R, lo hace en menor proporción que los aditivos SP1, SP2 y SP3. La incorporación de un $0,3 \%$ del aditivo SP4 a las pastas de cemento CEM III/B $32.5 \mathrm{R}$ no produce su segregación al contrario de lo observado en los otros 3 aditivos, incrementando aproximadamente en un $23,5 \%$ su fluidez. En el caso de las pastas del cemento de CAC, la adición de un 0,1\% de SP4 incrementa un $87 \%$ la fluidez, aunque experimentan una rápida pérdida de la misma, mientras para dosificaciones del $0,3 \%$ experimentan segregación.

3.1.2. Determinación de los parámetros reológicos mediante viscosímetro rotacional

A través de los ciclos de histéresis de las diferentes pastas de cemento obtenidos en un reómetro rotacional, se ha comprobado que en las ocho pastas de cemento el área de dicho ciclo de histéresis es muy pequeña y que, a excepción de las pastas del cemento tipo CEM I 52.5N/SR, la curva de incremento de la velocidad de cizalla prácticamente se superpone con la de descenso de la velocidad, lo que indica que las pastas están totalmente defloculadas.

Las ocho pastas de cemento presentan el mismo comportamiento reológico ya que en todos los casos las

\section{RESULTS}

\subsection{Rheological behaviour}

\subsection{1. "Minis/ump" test}

Minislump test results are shown in Figure 2. These results show that admixtures $S P 1, S P 2$, and $S P 3$ induced greater flowability than admixture SP4.

Admixtures SP1, SP2 and SP3 raised CEM I 42.5R, CEM I 52.5N/SR, CEM I 52.5R, CEM II/AV 42.5R and CEM II/BL $32.5 R$ cement pastes fluidity by $16-40 \%$ with dosages of $0.3 \%$, while BL I 52.5R paste fluidity was only increased by admixture SP3. Moreover, CEM III/B 32.5R and $C A C$ cement pastes segregated when a $0.3 \%$ dosage of SP1, SP2 or SP3 was used. A $0.1 \%$ dosage of these admixtures improved CEM III/B 32.5R cement paste fluidity by up to $53 \%$ and CAC paste flowability by up to 90\%, although this effect disappeared immediately in the latter when admixture SP1 or SP2 was used.

$S P 4$, on the contrary, barely modified the fluidity of CEM I 42.5R, CEM I 52.5R, CEM I 52.5N/SR, CEM II/AV 42.5R, CEM II/BL $32.5 R$ and BL I 52.5R cement pastes, and while it raised CAC and CEM III/B 32.5R fluidity, the effect was less intense than with SP1, SP2 and SP3. Unlike the other three admixtures, 0.3\% SP4 did not cause segregation in CEM III/B 32.5R cement pastes, while it raised its fluidity by approximately $23.5 \%$. With $0.1 \%$ SP4, CAC pastes gained $87 \%$ fluidity, although the effect disappeared quickly, whereas $0.3 \%$ doses caused segregation.

\subsubsection{Rotational viscosimeter results}

The rotational viscosimeter results showed that the area of the hysteresis cycles was very small in the eight pastes studied; moreover, with the exception of the CEM I 52.5N/SR pastes, the up-ramp shear rate curve practically coincided with the down-ramp curve, an indication that the pastes were wholly deflocculated.

The eight cement pastes exhibited the same rheological behaviour, for in all cases the down-ramp curves fitted a 


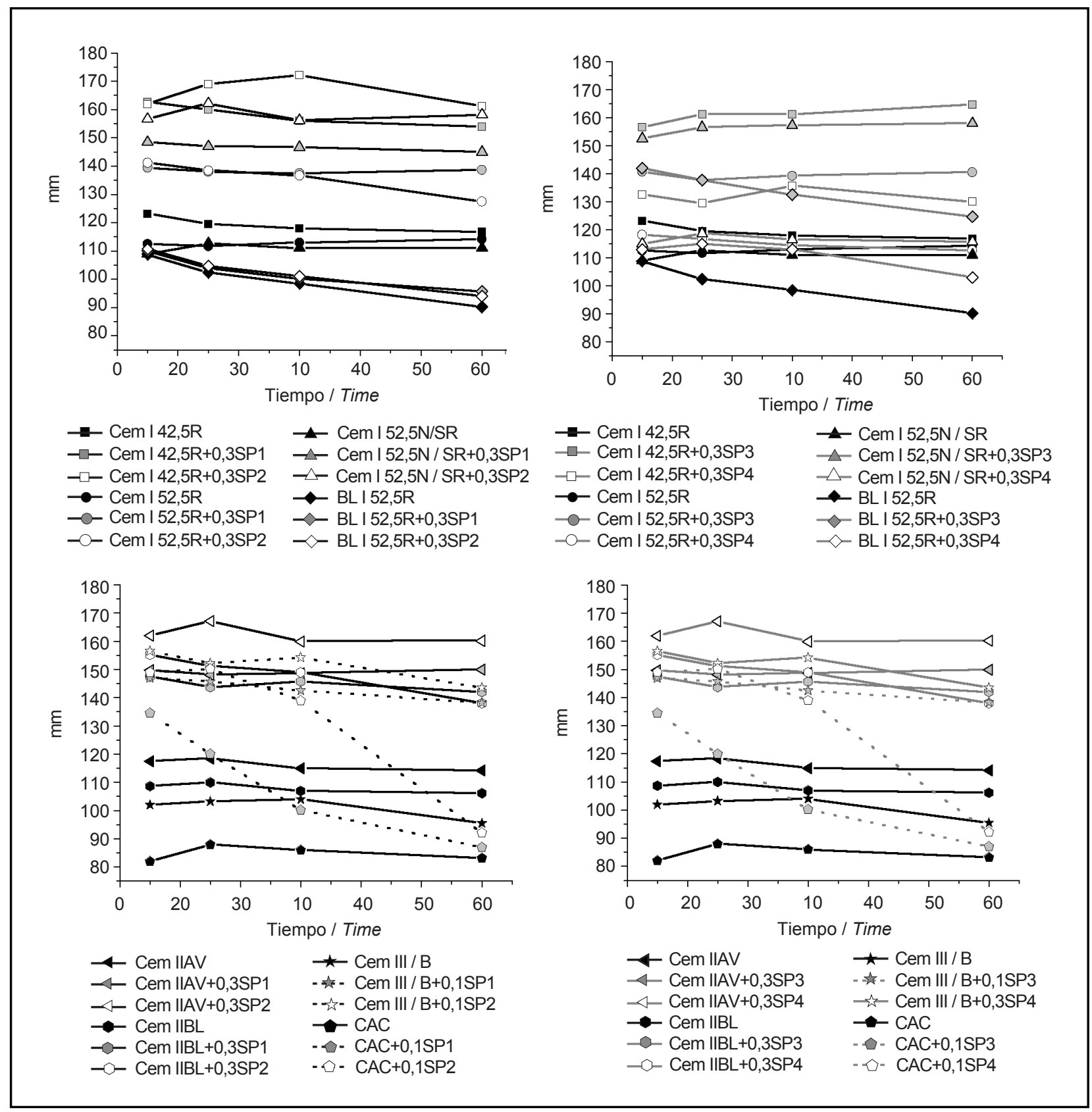

Figura 2. Evolución de la fluidez de las pastas de cemento aditivadas determinada mediante el ensayo de "Minislump". Figure 2. Minislump test results: variation in the fluidity of admixture-containing cement pastes.

descenso de la velocidad se ajustan a una línea recta de acuerdo a la ecuación de Bingham [1], cuya ordenada en el origen se asocia al esfuerzo de cizalla umbral y la pendiente a la viscosidad plástica de las pastas.

$$
\tau=\tau_{0}+\mu \dot{\gamma}
$$

Las Figuras 3 y 4 muestran la evolución de los parámetros reológicos, viscosidad plástica y esfuerzo de cizalla umbral, respectivamente, de las pastas de cemento en presencia de los diferentes aditivos superplastificantes straight line in keeping with the Bingham equation [1], in which the $y$-axis intercept indicates the yield shear stress and the slope, the plastic viscosity.

Figures 3 and 4 show the variation in the plastic viscosity and yield shear stress, respectively, of the cement pastes containing the superplasticizer admixtures studied. According to these figures, the admixtures 
estudiados. Dichas Figuras indican que los aditivos actúan de modo diferente dependiendo de sus características estructurales, de su dosificación y del tipo de cemento y tipo de adición que contiene el cemento.

Al igual que sucede en el ensayo de "Minislump", los aditivos SP1, SP2 y SP3 parecen tener un efecto similar sobre la fluidez de las distintas pastas de cemento y marcadamente superior al que muestra la incorporación del aditivo SP4.

La presencia de SP1, SP2 y SP3 reduce hasta $96 \%$ el valor del esfuerzo de cizalla umbral de las pastas de los cementos tipo CEM I 42.5R, CEM I 52.5N/SR y CEM II/AV 42.5R, con respecto a las correspondientes pastas sin aditivo, cuando se incorporan en una dosificación del 0,3\%. Esta reducción es más patente cuando los aditivos empleados son SP2 y SP3. El efecto de estos aditivos sobre este parámetro reológico es ligeramente inferior en el caso de las pastas de cemento de los tipos CEM I 52.5R y CEM II/BL 32.5R en la worked differently depending on their structural characteristics and dosage, as well as the type of cement and cement addition.

As in the minislump test results, admixtures SP1, SP2 and SP3 appeared to have a similar effect on cement paste flowability, and a substantially greater effect than admixture SP4.

At $0.3 \%$ dosages, SP1, SP2 and SP3 lowered the yield shear stress by up to $96 \%$ in CEM I 42.5R, CEM I 52.5N/SR and CEM II/AV 42.5R pastes. The reduction was more accentuated with admixtures SP2 and SP3. The effect of these admixtures on CEM I 52.5R and CEM II/BL $32.5 R$ pastes was slightly smaller, with decreases of $74 \%$ and $84 \%$, respectively. The $0.3 \%$ SP1- and SP2- mediated reduction of yield shear stress in white cement pastes was fairly small, $5.8 \%$ and

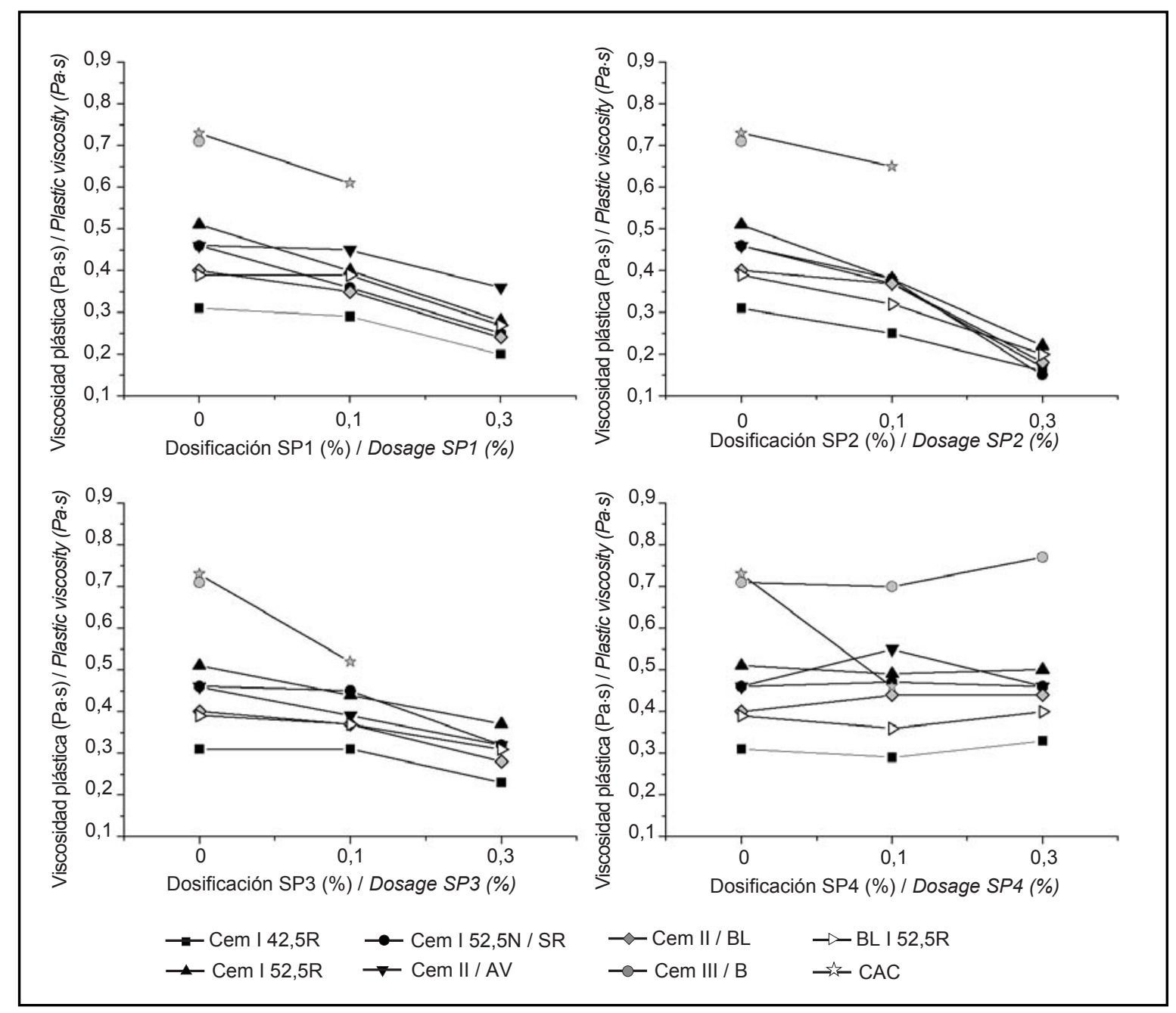

Figura 3. Evolución de la viscosidad plástica de las pastas de cemento aditivadas.

Figure 3. Variation in the plastic viscosity of cement pastes containing admixtures. 


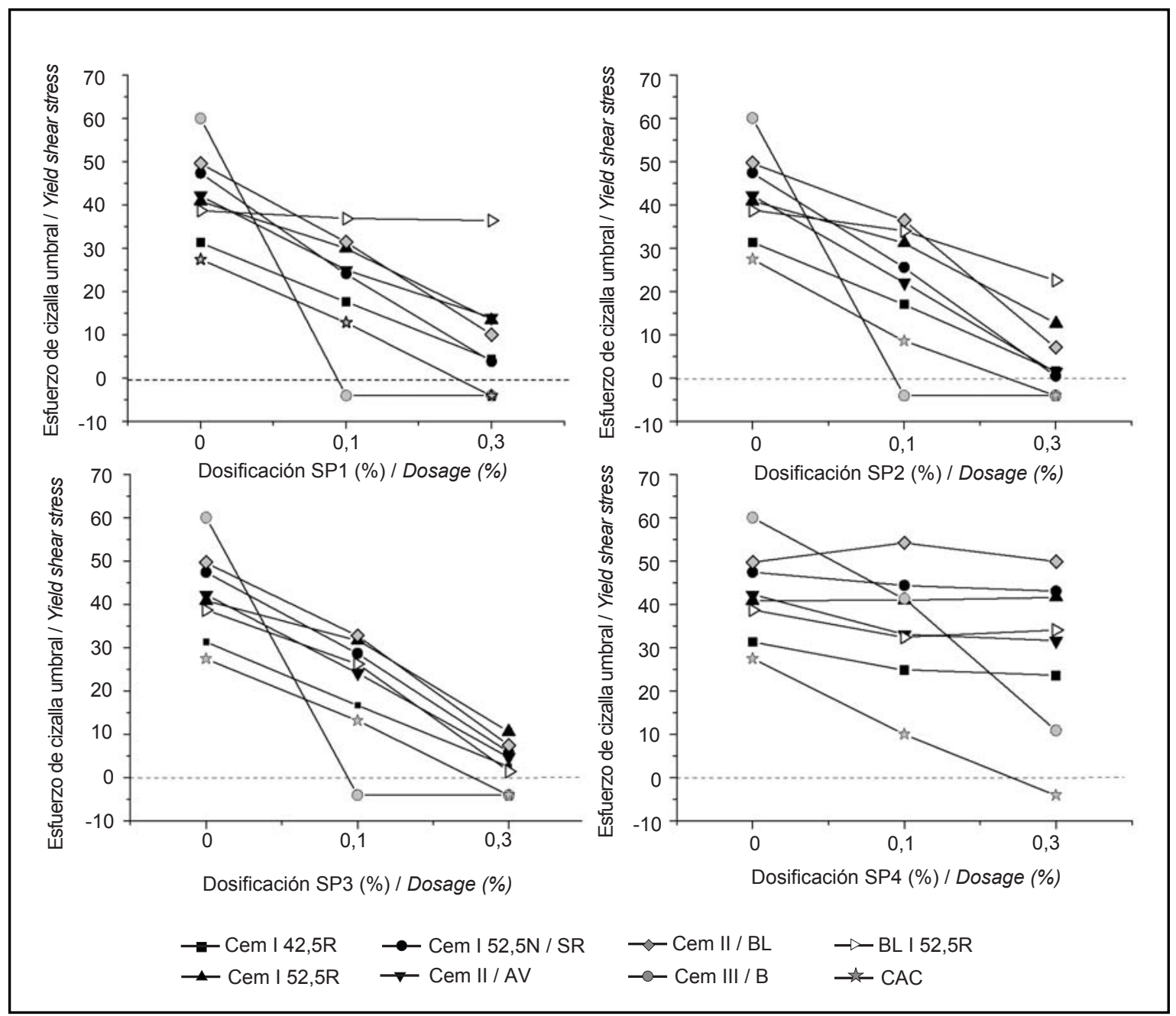

Figura 4. Evolución del esfuerzo de cizalla de las pastas de cemento aditivadas. Figure 4. Variation in shear stress in cement pastes containing admixtures.

que la reducción es de hasta el $74 \%$ y $84 \%$, respectivamente. La reducción que los aditivos SP1 y SP2 originan sobre el esfuerzo de cizalla umbral de las pastas de cemento blanco es muy baja siendo del $5,8 \%$ y $41,06 \%$, respectivamente, cuando su dosificación es del 0,3\%, mientras que la misma dosificación de SP3 reduce hasta un 96\% dicho parámetro. Por el contrario, y en similitud con los resultados observados mediante el ensayo de "Minislump", la mayor reducción que originan SP1, SP2 y SP3 sobre el esfuerzo de cizalla umbral se produce sobre las pastas de cemento tipo CEM III/B 32,5R y CAC, de forma que la incorporación de tan sólo un $0,1 \%$ de dichos aditivos sobre las primeras produce su segregación. En el caso de las pastas de CAC la incorporación de un $0,1 \%$ de SP2 induce una reducción de hasta el $68 \%$, mientras que dosificaciones del 0,3\% de SP1, SP2 y SP3 producen su segregación.

Finalmente, la incorporación del aditivo SP4 no ejerce ninguna influencia sobre el esfuerzo de cizalla umbral de las pastas de los cementos CEM I 52,5R, CEM I 52,5NSR
$41.06 \%$, respectively, whereas SP3 lowered this parameter by up to $96 \%$. Yield shear stress was reduced most effectively by SP1, SP2 and SP3 in CEM III/B $32.5 R$ and $C A C$ pastes, a finding consistent with the minislump test results; moreover, a dosage of $0.1 \%$ of these admixtures caused segregation in CEM III/B $32.5 R$. In CAC pastes the inclusion of $0.1 \%$ of $S P 2$ induced a reduction of up to $68 \%$, whereas $0.3 \%$ dosages of SP1, SP2 and SP3 caused segregation.

Finally, the inclusion of admixture SP4 appeared to have no effect on yield stress in CEM I 52.5R, CEM I 52.5N/SR or CEM II/BL cement pastes. In CEM I 42.5R, CEM II/AV 
y CEM II/BL. En el caso de pastas de los cementos CEM I 42.5R, CEM II/AV 42.5R y BL I 52.5R, dosificaciones del $0,1 \%$ reducen en torno a un $15 \%-20 \%$ el esfuerzo de cizalla umbral, no detectándose un mayor descenso de dicho parámetro cuando la dosificación se incrementa al $0,3 \%$. Finalmente, la influencia del aditivo SP4 es máxima en el caso de los cementos CEM III/B 32.5R y CAC, originando con dosificaciones del $0,3 \%$ en el primer caso una reducción de hasta el $82 \%$ del esfuerzo de cizalla umbral y en el segundo su segregación.

Con respecto al efecto de los diferentes aditivos sobre la viscosidad plástica, la Figura 4 muestra que el aditivo SP2 es el que disminuye más significativamente dicho parámetro, llegando a ser dicha reducción de hasta el $65 \%$ cuando se incorpora un $0,3 \%$ de dicho aditivo a las pastas de los cementos tipo CEM II/AV 42.5R y CEM I 52.5N/SR. Por el contrario, el aditivo SP4, a ninguna de las dosificaciones estudiadas, modifica el valor de la viscosidad plástica de las diferentes pastas con respecto a aquellas que carecen de él, a excepción de las pastas de cemento CAC, las cuales experimentan una reducción del $37 \%$ de dicho parámetro reológico cuando se incorpora un $0,1 \%$ de SP4.

\subsection{Determinación del potencial zeta}

El valor del potencial zeta de cada uno de los cementos estudiados se muestra en la Tabla 5 . Se observa que todos los cementos empleados presentan un potencial zeta positivo siendo hasta 7 veces superior el correspondiente al cemento CAC. Con respecto al potencial zeta de las adiciones, se observa que dicho potencial es positivo en el caso de las cenizas volantes, mientras que la escoria y la caliza presentan potenciales zeta negativos.

\section{DISCUSIÓN}

De acuerdo con los ensayos reológicos realizados, se deduce que todas las pastas de cemento estudiadas se comportan como fluidos de Bingham de forma que en los ciclos de histéresis la curva de descenso de la velocidad de cizalla se ajusta a una recta definida por la ecuación [1] (29). La incorporación de aditivos basados en policarboxilato y poliéteres no modifica el modelo reológico al que se ajustan dichas pastas, comportándose todas ellas a su vez como fluidos de Bingham. Sin embargo, la presencia de dichos
42.5R and BL I 52.5R cement pastes, 0.1\% dosages lowered the yield stress by $15-20 \%$, although this parameter declined no further when the dosage was raised to $0.3 \%$. Finally, the effect of admixture SP4 was greatest in CEM III/B 32.5R and CAC cements, lowering yield stress by up to $82 \%$ in the former and causing segregation in the latter when the dosage was $0.3 \%$.

Figure 4, in turn, shows that SP2 was the admixture that lowered plastic viscosity most, by up to $65 \%$ at dosages of $0.3 \%$, in CEM II/AV $42.5 R$ and CEM I $52.5 \mathrm{~N} / \mathrm{SR}$ pastes. By contrast, SP4 did not modify the plastic viscosity of the pastes at any of the dosages studied, with the exception of the CAC, for which the value declined by $37 \%$ with a $0.1 \%$ dosage of the admixture.

\subsection{Zeta-potential}

The zeta-potential values for the cements studied are given in Table 5. All the cements exhibited a positive zeta-potential, with values up to seven times higher for CAC. The zeta-potential of the additions, in turn, was positive for fly ash, but negative for slag and limestone.

\section{DISCUSSION}

According to the rheological tests conducted, all the pastes behaved like Bingham fluids, due to in the respective hysteresis cycles, the down-ramp of the shear rate curve fit the straight line defined by equation [1] (29). The inclusion of polycarboxylate and polyether admixtures did not modify the rheological model followed by these pastes, all of which likewise behaved like Bingham fluids. The presence of the admixtures did change the value of the rheological parameters, however, particularly

Tabla 5 / Table 5

Potencial zeta de los cementos

Cement zeta-potential

\begin{tabular}{|lccccccccccc|}
\hline $\begin{array}{l}\text { Tipo de cemento } \\
\text { Cement }\end{array}$ & Cem I & Cem I & Cem I & Cem II & Cem II & Cem III & BL I & CAC & Ceniza & Escoria & $\begin{array}{c}\text { Caliza } \\
\text { Limestone }\end{array}$ \\
\hline $\begin{array}{l}\text { Potencial Zeta }(\mathrm{mV}) \\
\text { Zeta-potential }\end{array}$ & +1.6 & +1.5 & +1.3 & +1.5 & +1.8 & +1.4 & +1.8 & +10.4 & +1.7 & -2.9 & -8.5 \\
\hline
\end{tabular}


aditivos sí modifica el valor de sus parámetros reológicos, fundamentalmente del esfuerzo de cizalla umbral, observándose que el efecto de los aditivos basados en policarboxilato y poliéteres depende fundamentalmente de la estructura de dichos aditivos y del tipo de cemento utilizado.

Con respecto a la influencia de las características estructurales de los aditivos, se observa que el número de grupos GC y el número de grupos GE (ver Tabla 3) son factores determinantes sobre el incremento de la fluidez y la modificación de los parámetros reológicos de las pastas de cemento estudiadas. En general, los resultados de "Minislump" y de esfuerzo de cizalla umbral coinciden en que los aditivos SP1, SP2 y SP3 ejercen un mayor incremento de la fluidez sobre todas las pastas de cemento con respecto al inducido por el aditivo SP4. Esta significativa mejora de las propiedades reológicas se debe, por una parte, a que los aditivos SP1, SP2 y SP3 presentan un mayor contenido de grupos GC (ver Tabla 3), de forma que se produce una mayor adsorción del aditivo sobre las partículas de cemento que en el caso del aditivo SP4 con un contenido de grupos GC significativamente menor $(4,7 \%)$. Por otro lado, el contenido de grupos GE y, por tanto, la longitud de las cadenas laterales de poliéteres determinan la repulsión estérica inducida por el aditivo. Los resultados obtenidos en los ensayos reológicos realizados confirman que los aditivos SP2 y SP3 con un contenido de grupos GE de $69,4 \%$ y $78,3 \%$, respectivamente, inducen un mayor efecto fluidificante $(3,7)$. Por el contrario, el aditivo SP4, a pesar de la elevada longitud y número de cadenas laterales que lo constituyen, no origina, en la mayoría de los casos estudiados, un significativo incremento de la fluidez ni una modificación importante de los parámetros reológicos de las pastas de cemento. Esto es debido a que la cantidad de grupos GC que contiene es muy pequeña $(4,7 \%)$, siendo la adsorción del aditivo SP4 sobre las partículas de cemento menor que la de los aditivos SP1, SP2 y SP3, y consecuentemente, menor su efecto fluidificante.

Con respecto a la influencia de las características del cemento sobre el incremento de la fluidez inducida por los aditivos, se observa que son factores determinantes sobre dicho efecto su composición química y mineralógica, su finura y distribución de tamaño de partícula, su contenido de $\mathrm{C}_{3} \mathrm{~A}$, las relaciones $\mathrm{C}_{3} \mathrm{~A}$ /sulfato cálcico $\mathrm{y}$ $\mathrm{C}_{3} \mathrm{~S} / \mathrm{C}_{3} \mathrm{~A}$, así como el tipo de adición que contenga.

En las pastas de cemento tipo CEM I 42.5R y CEM I 52.5R con muy similar composición química y mineralógica, la modificación de los parámetros reológicos, así como el incremento de la fluidez determinada mediante el ensayo de "Minislump" que los diferentes aditivos inducen, es mayor en pastas cemento del tipo CEM I 42.5R con respecto a las del cemento CEM I 52.5R. Esto es debido a que la superficie específica del cemento CEM I 52.5R es mayor the yield stress. This effect of polycarboxylate and polyether superplasticizers depended essentially on admixture structure and the type of cement.

Hence, the number of $P C$ and PE groups in the admixture structure (see Table 3) was found to be a determining factor for increased fluidity and the modification of paste rheological parameters. Overall, the minislump and yield stress results concurred that admixtures SP1, SP2 and SP3 had a more intense effect than SP4 on the fluidity of all the cement pastes. This significant improvement in rheological properties was due on the one hand to the fact that admixtures SP1, SP2 and SP3 exhibited a higher CG content (see Table 3), providing for greater admixture adsorption on the cement particles than observed for SP4, which had a significantly smaller CG content (4.7\%). Moreover, the EG content and therefore the length of the polyether lateral chains determines the steric repulsion induced by the admixture. The results obtained in the rheological tests confirmed that admixtures SP2 and SP3, with EG contents of $69.4 \%$ and $78.3 \%$, respectively, were more effective fluidizers $(3,7)$. On the contrary, admixture SP4, despite the large number of lengthy chains in its structure, failed in most cases to generate any significant increase in fluidity or substantial change in cement paste rheological parameters. This was due to its small CG content $(4.7 \%)$, which determined its lower rate of adsorption on cement particles and concomitantly less intense fluidizing effect than found for admixtures SP1, SP2 and SP3.

Cement characteristics, in turn, such as chemical and mineralogical composition, fineness and particle size distribution, $C_{3} A$ content, $C_{3} A$ /calcium sulphate and $C_{3} S / C_{3} A$ ratios and the type of addition, were observed to be determinants in the increased fluidity induced by the admixtures.

While CEM I 42.5R and CEM I 52.5R had a very similar chemical and mineralogical composition, the modification of rheological parameters induced by the various admixtures, as well as the increased fluidity found with the minislump test, were greater in CEM I 42.5R than CEM I 52.5R pastes. This is because, with a larger specific surface area, cement CEM I 52.5R (481.5 m²/g compared to $386.7 \mathrm{~m}^{2} / \mathrm{g}$ for CEM I 42.5R) needs a 
$\left(481,5 \mathrm{~m}^{2} / \mathrm{g}\right)$ que la del CEM I 42.5R $\left(386,7 \mathrm{~m}^{2} / \mathrm{g}\right)$, siendo necesario, en el primer caso, una mayor dosificación de aditivo para obtener un efecto fluidificante similar $(16,19,30-31)$.

En las pastas de cemento sin adición, la diferente composición mineralógica y principalmente el contenido en $\mathrm{C}_{3} \mathrm{~A}$, así como las relaciones $C_{3} A$ /sulfato cálcico y $C_{3} S / C_{3} A$, son los parámetros que más influyen en el efecto fluidificante inducido por los aditivos. De acuerdo con la bibliografía (17), la fase $C_{3} A$ es la fase mineralógica del cemento que tiene una mayor capacidad de adsorción de los aditivos basados en policarboxilatos debido a que presenta valores de potencial zeta más positivos que las fases $\mathrm{C}_{3} \mathrm{~S}$ y $\mathrm{C}_{2} \mathrm{~S}$. Si el aditivo es adsorbido preferiblemente sobre la fase $\mathrm{C}_{3} \mathrm{~A}$, queda menor cantidad de aditivo en la fase acuosa disponible para adsorberse sobre las fases silicato y ejercer de esta forma una repulsión entre ellas. En presencia de yeso, los aditivos y los sulfatos compiten por la fase $C_{3} A$, siendo más favorable la reacción con los sulfatos y formándose preferiblemente etringita. De esta forma, queda más aditivo disponible en la fase acuosa para ejercer un efecto fluidificante en las pastas. Por lo tanto, el contenido de $C_{3} A$ del cemento, así como la relación $C_{3} A$ /sulfato cálcico, tienen una influencia directa sobre el comportamiento fluidificante de los aditivos, siendo dichos valores fundamentales para explicar el diferente efecto de los aditivos sobre las pastas de cemento tipo CEM I $52.5 \mathrm{~N} / \mathrm{SR}$ y BL I 52.5R. El cemento tipo CEM I $52.5 \mathrm{~N} / \mathrm{SR}$, al ser un cemento resistente a los sulfatos, presenta un contenido en $\mathrm{C}_{3} \mathrm{~A}$ muy bajo $(0,5 \%)$, aunque el contenido en sulfatos es elevado. De esta forma, la relación $\mathrm{C}_{3} \mathrm{~A} /$ sulfatos es muy baja, mientras que la relación $C_{3} S / C_{3} A$ es elevada (ver Tabla 6) y todo el $C_{3} A$ reacciona con el yeso para formar etringita, quedando aditivo disponible en disolución para ejercer el efecto dispersante observado en los ensayos reológicos. Por el contrario, el cemento tipo BL I 52.5R presenta un elevado porcentaje en $\mathrm{C}_{3} \mathrm{~A}(7,7 \%)$ y aunque la relación $\mathrm{C}_{3} \mathrm{~A} /$ sulfatos es elevada y la relación $\mathrm{C}_{3} \mathrm{~S} / \mathrm{C}_{3} \mathrm{~A}$ es baja, quedaría fase $\mathrm{C}_{3} \mathrm{~A}$ sin reaccionar con los sulfatos capaz de intercalar polímero entre las láminas de su estructura, pudiendo formar una fase órgano-mineral (32). Consecuentemente, en las pastas de cemento BL I 52.5R el aditivo podría ser consumido en gran medida por la fase aluminato $y$, por lo tanto, poco adsorbido sobre las fases silicato, lo cual explicaría el bajo poder fluidificante de los aditivos sobre dicho cemento, lo que concuerda con el bajo efecto dispersante observado en los ensayos reológicos realizados.

Con respecto a las pastas de cemento que contienen adiciones, se observa que el tipo de adición ejerce un papel fundamental en el comportamiento fluidificante de los aditivos. Cuando la adición es caliza (CEM II/BL 32.5R), el efecto dispersante de todos los aditivos es comparable al producido sobre las pastas de CEM 42.5R (Ver Figuras higher dosage of admixture to obtain a similar fluidizing effect $(16,19,30-31)$.

In non-blended cement pastes, mineralogical composition and primarily the $C_{3} A$ content, as well as the $C_{3} A$ /calcium sulphate and $C_{3} S / C_{3} A$ ratios, were the parameters with the greatest effect on admixtureinduced fluidity. Pursuant to the literature (17), phase $C_{3} A$ is the cement mineralogical phase with greatest capacity to adsorb polycarboxylate admixtures, due to its zeta-potential values are more positive than the $C_{3} S$ and $C_{2} S$ values. If the admixture is more readily adsorbed on the $C_{3} A$, smaller amounts are available in the aqueous phase to adsorb on silicate phases, generating their repulsion. When gypsum is present in the medium, the admixtures and sulphates compete for the $C_{3} A$, but the sulphates adsorption is more favorable and form ettringite with $C_{3} A$. As a result, more admixture is available in the aqueous phase to enhance paste fluidity. The $C_{3} A$ content and the $C_{3} A$ /calcium sulphate ratio in the cement therefore have a direct effect on admixture effectiveness and essentially explain the differential effect of the admixtures on CEM I 52.5N/SR and BL I 52.5R cement pastes. Cement CEM I $52.5 \mathrm{~N} / S R$, as a sulphate-resistant cement, has a very low $C_{3} A$ content $(0.5 \%)$, although its sulphate content is high. Hence, the $C_{3} A$ /sulphates ratio is very low, whereas the $C_{3} S / C_{3} A$ ratio is high (see Table 6) and all the $C_{3} A$ reacts with the gypsum to form ettringite, leaving admixture in the solution to cause the dispersive effect observed in the rheological tests. On the contrary, BL I $52.5 R$ cement has a high percentage of $C_{3} A(7.7 \%)$ and inasmuch as the $C_{3} A$ /sulphate ratio is high and the $C_{3} S / C_{3} A$ ratio is low, the remaining $C_{3} A$ that does not react with the sulphates could intersperse polymers in between the layers of its structure, forming an organicmineral phase (32). Consequently, in BL I 52.5R cement pastes, the admixture may be largely consumed by the aluminate phase and therefore scantly adsorbed on the silicate phases. This would explain the low fluidizing power of the admixtures in this cement, which concurs with the low degree of dispersion observed in the rheological tests conducted.

In blended-cement cement pastes, the type of addition was found to play an essential role in the admixture effectiveness. When the addition was limestone (CEM $I I / B L 32.5 R)$, the dispersive effect of all the admixtures was comparable to the effect in CEM 42.5R pastes (see Figures 2 and 4). On the contrary, Björnstrom and 
Tabla 6 / Table 6

Relaciones $\mathrm{C}_{3} \mathrm{~A} /$ Sulfato cálcico y $\mathrm{C}_{3} \mathrm{~S} / \mathrm{C}_{3} \mathrm{~A}$ de los diferentes cementos

Relationship between $C_{3} A$ /calcium sulphate and $C_{3} S / C_{3} A$ in the cements

\begin{tabular}{|l|ccc|}
\hline & $\begin{array}{c}\text { Cem I } \\
\mathbf{5 2 , 5 N} / \mathbf{S R}\end{array}$ & $\begin{array}{c}\text { Cem I } \\
\mathbf{4 2 , 5 R}\end{array}$ & $\begin{array}{c}\text { BL I } \\
\mathbf{5 2 , 5 R}\end{array}$ \\
\hline $\mathrm{C}_{3} \mathrm{~A} /$ sulfatos cálcicos & 0.15 & 0.55 & 1.6 \\
$\mathrm{C}_{3} \mathrm{~A} /$ calcium sulphate & & & \\
\hline $\mathrm{C}_{3} \mathrm{~S} / \mathrm{C}_{3} \mathrm{~A}$ & 132.8 & 15.62 & 8.5 \\
\hline
\end{tabular}

2 y 4). Por el contrario, Björnstrom y Chandra (9) indicaron que la presencia de aditivos superplastificantes inducían una menor disminución del esfuerzo de cizalla y de la viscosidad plástica en cementos con incorporación de caliza, con respecto a aquellos que carecen de esta adición. Dichos autores explicaron este menor efecto dispersante de los aditivos basados en policarboxilato en base a la mayor finura de dicha caliza y en base al diferente mecanismo de hidratación de estos cementos con respecto a aquellos que carecen de esta adición. El valor del potencial zeta de la caliza mostrado en la Tabla $5(-8,5$ $\mathrm{mV}$ ) no explicaría el comportamiento reológico observado de las pastas de cemento CEM II/BL 32.5R aditivadas, siendo necesarios más estudios en este sentido.

En las pastas de cemento CEM II/AV 42.5R, cuya adición es la ceniza volante, el efecto dispersante de los aditivos es similar al inducido sobre pastas de cemento sin adición tipo CEM I 42.5R. Este resultado se puede explicar en base a que el potencial zeta de una ceniza es positivo, pudiendo ésta adsorber también aditivo y producir la repulsión tanto entre los granos de cemento, como entre las partículas de ceniza. Además, la morfología esférica de las partículas de ceniza volante pudiera ejercer un efecto de reducción de las fricciones entre partículas y reduciría la demanda de agua (33).

Cuando la adición es escoria granulada de horno alto (cemento tipo CEM III/B 32.5R), todos los aditivos inducen una disminución muy significativa del esfuerzo umbral, ya a dosificaciones del $0,1 \%$, llegando incluso a producir segregaciones en las pastas con los aditivos SP2 y SP3. Resultados similares se han referido en trabajos previos (34). Esto parece indicar que la escoria (presente en un contenido superior al $70 \%$ en estos cementos) adsorbe parte del aditivo, produciéndose una repulsión tanto entre las partículas de escoria, como entre los granos de cemento.

Finalmente, los ensayos reológicos realizados demuestran que el mayor efecto fluidificante inducido por los aditivos se produce sobre las pastas de cemento de aluminatos de cálcico (CAC). En este caso, la evolución del esfuerzo de cizalla umbral de las pastas aditivadas es muy similar para los cuatro aditivos, produciendo segregación en todos los casos a dosificaciones del 0,3\%. Este mayor efecto de los aditivos sobre las propiedades reológicas del cemento CAC
Chandra (9) reported that the presence of superplasticizer admixtures induced a smaller reduction in yield stress and plastic viscosity in limestone blended cements than in those without this addition. These authors attributed the lower dispersive power of polycarboxylate admixtures to the lesser fineness of the limestone and to the difference in the hydration mechanisms in these cements compared to cements not containing this addition. The value of the zeta-potential of the limestone given in Table $5(-8.5 \mathrm{mV})$ would not explain the rheological behaviour observed in CEM II/BL 32.5R cement pastes. Further study is required in this regard.

In fly ash blended cement, CEM II/AV 42.5R, the dispersive effect of the admixtures was similar to the effect induced in CEM I 42.5R pastes. This result may be explained by the positive zeta-potential of fly ash, indicative of its ability to adsorb admixture, with the resulting repulsion among both the cement grains and the fly ash particles. Moreover, the spherical morphology of the fly ash particles may reduce the friction between particles, likewise lowering water demands (33).

When the addition was granulated blast furnace slag (CEM III/B 32.5R cement), all the admixtures induced a very significant reduction in yield stress, even at $0.1 \%$ dosages, and in fact admixtures SP2 an SP3 caused paste segregation at that dosage. Similar results were reported in previous papers (34). This would appear to indicate that the slag (with a content of over $70 \%$ in these cements) adsorbs part of the admixture, generating repulsion both between slag and cement particles.

Finally, the rheological tests conducted showed that the greatest fluidizing effect induced by the admixtures was on calcium aluminate cement (CAC) pastes. In this case, the variation in the yield stress in the pastes with admixtures was similar for all four substances, with segregation appearing at dosages of $0.3 \%$. This enhanced admixture effect on the rheological properties of CAC compared to the effect on Portland cement can be explained on the 
con respecto a las de cemento Portland se explica en base a su diferente composición mineralógica, basada fundamentalmente en aluminato monocálcico, siendo el potencial zeta de estos cementos más positivo $(+10.4 \mathrm{mV})$. Este potencial zeta más positivo favorece la adsorción de aditivo sobre las partículas de aluminato monocálcico (CA), siendo mayor el efecto dispersante ejercido.

\section{CONCLUSIONES}

Los resultados obtenidos tras el estudio realizado han permitido concluir que:

1. Las características estructurales de los aditivos estudiados que afectan principalmente al efecto fluidificante que éstos inducen son su contenido de grupos carboxilatos (GC), así como de grupos poliéteres (GE). El aditivo SP4, al presentar el menor contenido de grupos GC $(4,7 \%)$, apenas modifica las propiedades reológicas de las pastas de cemento, como consecuencia de su baja adsorción sobre los granos de cemento. Por el contrario, los aditivos SP1, SP2 y SP3, al presentar mayores contenidos de grupos GC, se absorben sobre las partículas de cemento, incrementando la fluidez de las pastas como consecuencia de la repulsión estérica ejercida por las cadenas laterales de grupos GE.

2. En relación con las características y composición de los cementos se deduce que:

- Cementos con similar composición química y mineralógica requieren mayor dosificación de aditivo para obtener un efecto fluidificante similar cuanto mayor es su finura.

- Entre los cementos sin adición, el CEM I 52.5N/SR es el que experimenta el mayor incremento de la fluidez en presencia de los aditivos como consecuencia de su baja relación $\mathrm{C}_{3} \mathrm{~A} /$ sulfato cálcico y su elevada relación $\mathrm{C}_{3} \mathrm{~S} / \mathrm{C}_{3} \mathrm{~A}$. En el extremo opuesto, la incorporación de aditivos a las pastas de BL I 52.5R origina la menor modificación de su fluidez debido a su alto contenido en $\mathrm{C}_{3} \mathrm{~A}$.

- En los cementos con adiciones el comportamiento de las pastas de cemento en presencia de aditivos es diferente, dependiendo de la naturaleza de la adición. En el caso de pastas de cemento CEM II/BL 32.5R con adición de caliza y CEM II/AV 42.5R con ceniza volante, el efecto de los aditivos es similar al inducido en pastas de cemento sin adición. Por último, las pastas de CEM III/B 32.5R, con escoria granulada de horno alto, son las que presentan incrementos de la fluidez más acusados, incluso a dosificaciones del 0,1\%.

- En pastas de cementos de aluminato de calcio se produce la mayor reducción de los parámetros reológi$\cos$, aunque experimentan una rápida pérdida de la fluidez. grounds of the differences in their mineralogical composition, particularly the high monocalcium aluminate content in CAC. This affords this type of cement a higher zeta-potential ( $+10.4 \mathrm{mV})$, favouring admixture adsorption on monocalcium aluminate (CA) particles, for a greater dispersive effect.

\section{CONCLUSIONS}

The following conclusions may be drawn from the results obtained in this study:

1. The structural characteristics of the admixtures studied that have the greatest effect on their fluidizing power are their carboxylate (CG) and polyether (EG) group content. Admixture SP4, with the lowest CG content $(4.7 \%)$, barely modified cement paste rheological properties, due to its low rate of adsorption on cement grains. On the contrary, admixtures SP1, SP2 and SP3, with higher CG contents, were adsorbed on the cement particles, increasing paste fluidity as a result of the steric repulsion generated by the lateral EG chains.

2. The deductions drawn in connection with cement characteristics and composition are:

- In cements with similar chemical and mineralogical composition, the greater the fineness, the higher the dosage of admixture required.

- CEM I 52.5N/SR was the cement without addition wich shows the highest rise in fluidity in presence of admixtures due to its low $C_{3} A$ /calcium sulphate and high $C_{3} S / C_{3} A$ ratios. The lowest modification in fluidity due to admixtures, in turn, was recorded for $B L I$ $52.5 R$ pastes due to their high $C_{3} A$ content.

- The effect of admixtures on blended cements varied depending on the nature of the addition. In the case of CEM II/BL 32.5R cement pastes, whose addition is limestone, and CEM II/AV 42.5R pastes, containing fly ash, the admixture-induced changes were similar to the modifications found for non-blended cement pastes. Lastly, CEM III/B 32.5R pastes, with granulated blast furnace slag additions, showed the highest rises in fluidity, even at dosages as small as $0.1 \%$.

- The greatest improvement in rheological parameters was found for calcium aluminate cement pastes, although the fluidity induced was observed to decline quickly. 


\section{AGRADECIMIENTOS}

Los autores agradecen al Ministerio de Educación y Ciencia la concesión del proyecto CTM2004-06619-CO2-01. También desean expresar su agradecimiento a A. Gil por su colaboración en la realización de los ensayos de "Minislump" y a J. J. Gaitero y A. Porro, de la Unidad Asociada CSIC/Labein-Tecnalia, por su cooperación en las diferentes medidas del potencial zeta.

\section{ACKNOWLEDGEMENTS}

This research was funded under Ministry of Education and Science grant CTM2004-06619-CO2-01. The authors wish to thank $A$. Gil for the assistance provided in the minislump test and J. J. Gaitero and A. Porro of the CSIC/Labein-Tecnalia Associate Unit for their cooperation in the zeta-potential measurements.

\section{BIBLIOGRAFÍA / BIBLIOGRAPHY}

(1) Valcuende, M. O., Parra, C. y Benlloch, J.: "Permeabilidad, porosidad y resistencia a compresión de hormigones autocompactables", Mater. Construcc., vol. 55, no 280 (2005), pp. 17-26.

(2) Martí Vargas, J. R., Serna-Ros, P., Arbeláez, C. A. y Rigueira-Víctor J. W.: "Comportamiento adherente del hormigón autocompactante en transición y anclaje", Mater. Construcc., vol. 56, n 284 (2006), pp. 28-42.

(3) Ohta, A., Sugiyama, T. y Tanaka, Y.: "Fluidizing mechanism and application of polycarboxylate-based superplasticizers", 5th CANMET/ACI (1997), pp. 359-378.

(4) Uchikawa, H., Hanehara, S. y Sawaki, D.: "The role of steric repulsive force in the dispersion of cement particles in fresh paste prepared with organic admixuture", Cem. Concr. Res., no 27 (1997), pp. 37-50.

(5) Yamada, K., Takahashi, T., Hanehara, S. y Matsuhisa, M.: "Effects of the chemical structure on the properties of polycarboxylatetype superplasticizer", Cem. Concr. Res., no 30 (2000), pp. 197-207.

(6) Hamada, D., Sato, T., Yamato, F. y Mizunuma, T.: "Development of new superplasticizers and its application to self-compacting concrete", $6^{\text {th }}$ CANMET/ACI (2000), pp. 269-290.

(7) Ferrari, G., Cerulli, T., Clemente, P., Dragoni, M., Gamba, M. y Surico, F.: "Influence of carboxylic acid-carboxylic ester ratio of carboxylic acid ester superplasticiser on characteristics of cement mixtures", 6 $6^{\text {th }}$ CANMET/ACI (2000) (Nice), pp. 505-519.

(8) Magarotto, R., Torresan, I. y Zeminian, N.: "Influence of the molecular weight of polycarboxilate ether superplasticizers on the rheological properties of fresh cement pastes, mortar and concrete", XI ICCC (Durban) (2003), pp. 514-526.

(9) Björnstrom, J. y Chandra, S.: "Effect of superplasticizers on the rheological properties of cements", Materials and Structures, vol. 36 (2003), pp. 685-692.

(10) Sugiyama, T., Ohta, A. y Uomoto, T.: "The dispersing mechanism and applications of polycarboxilate-based superplasticizers", XI ICCC (Durban) (2003), pp. 560-568.

(11) Mäeder, U., Schober, I., Wombacher, F. y Ludirdja, D.: "Polycarboxylate polymers and blends in different cements", Cement, Concrete and Aggregates, vol. 26 (2004), pp. 110-114.

(12) Winnefeld, F., Becker, S., Pakusch, J. y Götz, T.: "Polymer structure/concrete property relations of HRWRA", Eighth CANMET/ACI Internacional Conference on Recent Advances in Concrete Technology, Supplementary Papers (2006), pp. 159-177.

(13) Maeder, U. y Schober, I.: "Performance of blends of polycarboxylate polymers in different cements", XI ICCC (Durban), South Africa (2003), pp. 504-513.

(14) Flatt, R. J. y Houst, Y.: "A simplified view on chemical effects perturbing the action of superplasticizers", Cem. Concr. Res., no 31 (2001), pp. 1169-1176.

(15) Yamada, K., Ogawa, S. y Hanehara, S.: "Controlling of the adsorption and dispersing force of polycarboxylate-type superplasticizer by sulfate ion concentration in aqueous phase", Cem. Concr. Res., no 31 (2001), pp. 375-383.

(16) Chandra, S. y Björnström, J.: "Influence of cement and superplasticizers type and dosage on the fluidity of cement mortars. Part I", Cem. Concr. Res., no 32 (2002), pp. 1605-1611.

(17) Yoshioka, K., Tazawa, W., Hawai, K. y Enohata, T.: "Adsorption characteristics of superplasticizers on cement component minerals", Cem. Concr. Res., no 32 (2002), pp. 1507-1513.

(18) Magarotto, R., Torresan I. y Zeminian, N.: "Effect of alkaline sulphates on the performance of superplasticizers", 11th ICCC (Durban), South Africa (2003), pp. 569-580.

(19) Houst, Y., Bowen, P. y Siebold, A.: "Some basic aspects of the interaction between cement and superplasticizers", Innovations and developments in Concrete Materials and Construction. Ed. R. K. Dhir, P. C. Hewlett, L. J. Csetenvi (2002), pp. 225-234.

(20) Chongzhi, L., Dongmin, W., Shaomin, S. y Jialong, C.: "The compatibility of polycarboxylate-type superplasticizers with cement", Journal of Wuhan University of Technology-Mater. Sci. Ed. (2005), pp. 95-98.

(21) Ramachandran, S., Malhotra, V. M., Jolicoeur, C. y Spiratos, N.: Superplasticizers: properties and applications in concrete, CANMET, Ottawa, Canadá, 1998. 
(22) Rietveld, H. M.: "A profile refinement method for nuclear and magnetic structures", J. Applied Crystallog, no 2 (1969), pp. 65-71. (23) De la Torre, A. G., Cabeza, A., Calvente, A., Bruque, S. y Aranda, M. A. G.: "Full phase analysis of Portland clinker by penetrating synchrotron powder diffraction", Analytical Chemistry, no 73, 2 (2001), pp. 151-156.

(24) De la Torre, A. G., Bruque, S. y Aranda, M. A. G.: "Rietveld quantitative amorphous content analysis", J. Applied Crystallog, no 34 (2001), pp. 196-202.

(25) De la Torre, A. G. y Aranda, M. A. G.: "Accuracy in Rietveld quantitative phase analysis of Portland cements", J. Applied Crystallog, no 36 (2003), pp. 1169-1176.

(26) Grzeszcyk, S. y Sudol, M.: "The influence of the new generation superplasticizers on the rheological properties of cement pastes", $11^{\text {th }}$ ICCC (Durban), South Africa (2003), pp. 727-735.

(27) Palacios, M., Sierra, C. y Puertas. F.: "Métodos y técnicas de caracterización de aditivos para el hormigón", Mater. Construcc., vol. 53, no 269 (2003), pp. 89-105.

(28) Kantro, D. L.: "Influence of Water-Reducing Admixtures on the Properties of Cement Paste- A Miniature Slump Test", Cement, Concrete and Aggregate, vol. 2, no 2 (1980), pp. 95-102.

(29) Shaughnessy, R. y Clark, P. E.: "The Rheological Behaviour of Fresh Cement Pastes", Cem. Concr. Res., no 18 (1988), pp. 327-341. (30) Hanehara, S. y Yamada, K.: "Interaction between cement and chemical admixture from the point of of cement hydration, adsorption behaviour of admixture and paste rheology", Cem. Concr. Res., no 29 (1999), pp. 1159-1165.

(31) Bonen, D. y Sarkar, S. L.: "The superplasticizer adsorption capacity of cement pastes, pore solution composition and parameters affecting flow loss", Cem. Concr. Res., no 25 (1995), pp. 1423-1434.

(32) Plank, J., Dai, Z., Zouaoui, N. y Vlad D.: "Intercalation of Polycarboxylate superplasticizers into $C_{3} A$ hydrate phases", $8^{\text {th }}$ CANMET/ACI Superplasticizers and other chemical admixtures in concrete, Sorrento (Italia) (2006), pp. 201-214.

(33) Ferraris, C. F., Obla, K. H. y Hill, R.: "The influence of mineral admixtures on the rheology of cement paste and concrete", Cem. Concr. Res., no 31 (2001), pp. 245-255.

(34) Puertas, F., Alonso, M. M., Vázquez, T.: "Influencia de aditivos basados en policarboxilatos sobre el fraguado y el comportamiento reológico de pastas de cemento Portland", Mater. Construcc., vol. 55, no 277 (2005), pp. 61-73. 